\title{
Long Head of the Biceps Tendon Tenotomy versus Subpectoral Tenodesis in Rotator Cuff Repair
}

\author{
Jangwoo Kim, MD, Ji Hoon Nam, MD, Yuna Kim, MS, Jong Seop Kim, MD, Sae Hoon Kim, MD \\ Department of Orthopedic Surgery, Seoul National University Hospital, Seoul National University College of Medicine, Seoul, Korea
}

Background: Lesions of the long head of the biceps tendon (LHBT) are one of the most common pathologies in patients with a rotator cuff tear. Although various procedures have been shown to be effective for treating LHBT lesions during rotator cuff repair, no consensus has been reached regarding the most effective treatment. The purpose of this study was to compare the outcomes of tenotomy vs subpectoral tenodesis of the LHBT in arthroscopic rotator cuff repair.

Methods: The records of 135 patients who underwent arthroscopic rotator cuff repair with biceps tenotomy or subpectoral tenodesis for a partial LHBT tear of $>50 \%$ were initially reviewed. Finally, 77 patients (38 patients with tenotomy and 39 patients with subpectoral tenodesis) with an intact rotator cuff, who underwent a functional evaluation at 1 year postoperatively, were enrolled in this retrospective study.

Results: The average follow-up was $13.3 \pm 4.36$ months (13.2 \pm 1.4 months in the tenotomy group and $13.6 \pm 2.7$ months in the subpectoral tenodesis group; $p=0.416$ ). Demographic and surgical data were not significantly different between the 2 groups. Preoperatively, biceps groove tenderness, Speed's test, and Yergason test results were positive in $27.3 \%, 27.3 \%$, and $10.4 \%$ of the study subjects, respectively. Compared with preoperative values, all functional scores including shoulder muscle power were significantly improved postoperatively, and no significant intergroup difference was observed (all $p>0.05$ ). A visible Popeye deformity was not encountered in either group at the final follow-up. Eight patients in the tenotomy group and 7 patients in the subpectoral tenodesis group complained of mild anterior shoulder pain $(p=0.731)$, and 4 patients in each group complained of groove tenderness $(p=0.969)$. No surgical or postoperative complication occurred in either group.

Conclusions: Both biceps tenotomy and subpectoral tenodesis performed during rotator cuff repair improved pain and function and resulted in comparable clinical outcomes. Residual symptoms associated with the remnant LHBT in the groove may not be a problem after adhesion of LHBT.

Keywords: Rotator cuff repair, Tenotomy, Subpectoral tenodesis, Popeye deformity, Hidden lesion

Many associated pathologies may be encountered in rotator cuff repair, and one of the most common is the lesions of the long head of the biceps tendon (LHBT). ${ }^{1)}$ Preoperatively, it is difficult to determine whether the patient has an LHBT lesion in the presence of a rotator cuff tear.

Received December 9, 2019; Accepted December 26, 2019

Correspondence to: Sae Hoon Kim, MD

Department of Orthopedic Surgery, Seoul National University Hospital, 101 Daehak-ro, Jongno-gu, Seoul 03080, Korea

Tel: +82-2-2072-3930, Fax: +82-2-764-2718

E-mail: drjacobkim@gmail.com
However, during rotator cuff repair, this concomitant pathology can be easily identified by arthroscopy and clinicians usually try to address it since it may affect clinical outcomes. ${ }^{2,3)}$ Lesions of the LHBT and surrounding tissues (e.g., partial tears, subluxation, or dislocation of LHBT and superior labral anterior and posterior [SLAP] lesions) and anterior or posterior biceps pulley tears (pulley lesions) are easily observed during rotator cuff repair. ${ }^{4-6)}$ Although various procedures have been shown to be effective for treating LHBT lesions during rotator cuff repair, ${ }^{7,8)}$ no consensus has been reached regarding the most effective treatment. 
Biceps tenotomy is a recognized, successful procedure, which is relatively straightforward and time-saving, and has been reported to reduce pain effectively. ${ }^{9,10)}$ However, Popeye deformity, cramping pain, and strength loss due to distal migration of the tendon are always of concern. Thus, tenodesis of the LHBT has been recommended by some authors. It preserves normal tension and power of the biceps, and thus is preferred in some settings such as in relatively young patients or individuals whose work involves heavy lifting. ${ }^{7,11)}$ Subpectoral tenodesis of the LHBT has recently gained in popularity because it could address "hidden lesions" of the LHBT in the bicipital groove as well as providing satisfactory outcomes. ${ }^{12)}$ This technique is considered to address bicipital pathologies and provide better outcomes.

In this study, we retrospectively analyzed and compared the outcomes of tenotomy and subpectoral tenodesis conducted in the presence of a partial LHBT tear at the time of rotator cuff repair. We hypothesized that subpectoral tenodesis would have better overall functional outcomes.

\section{METHODS}

This study was conducted using a retrospective, comparative design and was approved by the Institutional Review Board of Seoul National University Hospital (IRB No. H1909-048-1063).

\section{Inclusion and Exclusion Criteria}

Patients underwent concomitant rotator cuff repair and LHBT tenotomy or subpectoral tenodesis at our institute from December 2014 to November 2017. The following inclusion criteria were applied: (1) a full-thickness rotator cuff tear verified during arthroscopic rotator cuff repair, (2) biceps tenotomy or subpectoral tenodesis performed for the treatment of a partial LHBT tear (> 50\%), (3) an intact rotator cuff after repair, verified by magnetic resonance imaging (MRI; Sugaya grade 1-3) at 1 year postoperatively, and (4) functional evaluation at 1 year postoperatively. The exclusion criteria were revision surgery for a rotator cuff tear, partial-thickness rotator cuff tear, and complete LHBT rupture. There was no difference in indications between the tenotomy group and the subpectoral tenodesis group. The 2 procedures were performed consecutively at different time periods in patients with the same pathology. Tenotomy was used to treat patients with a partial LHBT tear from December 2014 to December 2015, and subpectoral tenodesis was used predominantly from January 2016 to November 2017. Of the 135 patients eligible, 41 (30.4\%) were not followed up adequately, and thus were excluded from the analysis. In addition, postoperative MRI showed that 11 patients in the tenotomy group and 6 patients in the subpectoral tenodesis group had an unhealed rotator cuff at 1 year postoperatively, and these patients were also excluded. Finally, 77 patients were enrolled.

\section{Surgical Technique and Group Allocation}

All surgical procedures were performed by a single surgeon (SHK). Surgery was performed under general anesthesia with no additional nerve block. All patients underwent all arthroscopic repair of a full-thickness rotator cuff tear using the single row repair technique in the lateral decubitus position.

An arthroscope was introduced to the glenohumeral joint through a posterior viewing portal, and intraarticular pathologies were examined carefully, especially the biceps status. Biceps procedures (135 cases), either biceps tenotomy or subpectoral tenodesis, were performed in patients with a biceps lesion, with a tear $>50 \%$, in the index period (Fig. 1). Tenotomy was performed using arthroscopic scissors to sever the biceps origin (Fig. 2). For subpectoral tenodesis, the LHBT was tagged with a suture to assist locating the tendon in the subpectoral area. In all cases, the proximal stump was secured in front of the hiatus of the bicipital groove; no complete distal migration occurred through the groove. Subpectoral tenodesis was conducted as follows: a longitudinal incision $(>2-3 \mathrm{~cm}$ in length) was made at the biceps, centered at the inferior margin of the pectoralis major tendon. Location of this

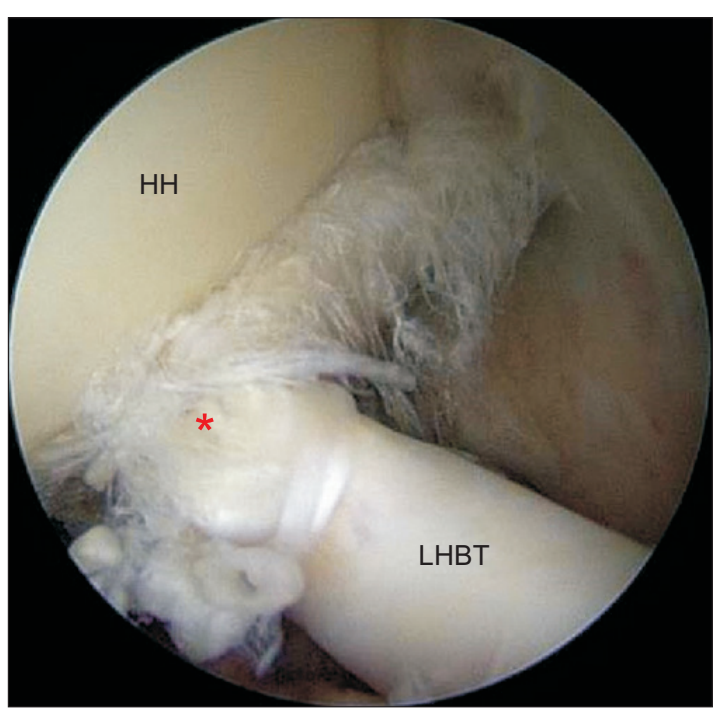

Fig. 1. Partial tear of the long head of the biceps tendon involving more than 50\% (asterisk). HH: humeral head, LHBT: long head of the biceps tendon. 


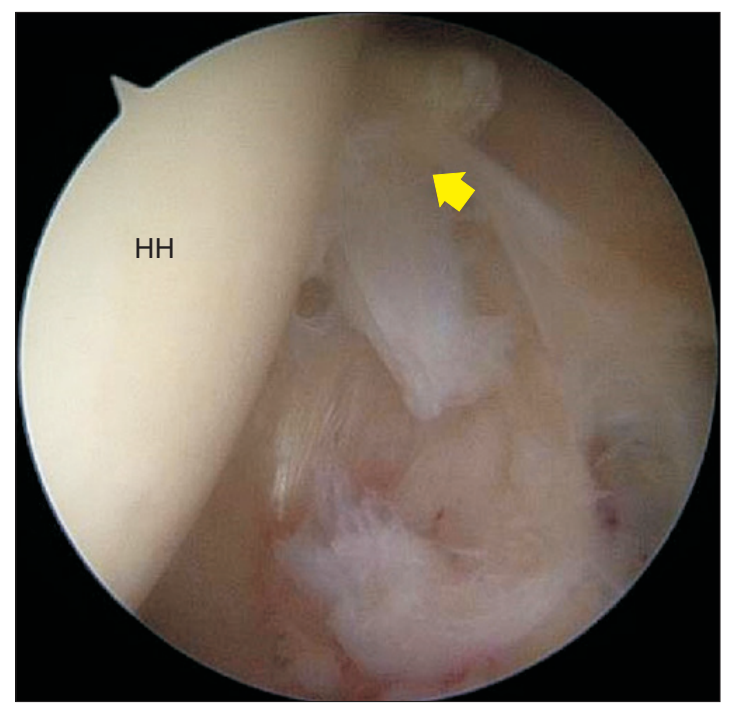

Fig. 2. After tenotomy, the biceps tendon (arrow) migrated distally, but it could be observed proximal to the bicipital hiatus. $\mathrm{HH}$ : humeral head.

incision was verified by muscle movement by pulling the previously placed LHBT tagging suture. After blunt dissection of the brachial fascia, the LHBT was easily found by superolateral retraction of the pectoralis major tendon sling. The LHBT was then pulled out (Fig. 3), fixed using a soft anchor (JuggerKnot; Biomet, Warsaw, IN, USA), and sutured using a lasso-loop stitch (Fig. 4). ${ }^{13)}$ Drilling and anchor insertion were performed with a guide in place directly underneath the pectoralis tendon at the tenodesis site. To maintain the length-tension relationship of the LHBT, it was sutured at the musculotendinous junction. ${ }^{14)}$

After tenotomy or tenodesis, the arthroscope was moved to the subacromial space. Selective acromioplasty was performed in a heel type spur or a lateral projecting spur. Rotator cuff repair was performed in a standardized manner in all study subjects. In each case, cuff footprints were prepared using a motorized burr to cause surface bleeding. Anchors were inserted at the lateral footprint margin through a new accessory portal created by a small stab incision. Triple loaded all-suture anchors, Y-Knot RC (ConMed Linvatec, Largo, FL, USA), were usually used. Loaded sutures were passed through the tendon at $0.5-1$ $\mathrm{cm}$ medial to its edge, using a flexible suture passer (Expressew; DePuy Mitek, Raynham, MA, USA). All knots were tied securely using a self-locking, sliding knot. The number of anchors used depended on the tear size: 1 anchor was used for small tears; $2-3$ anchors, for medium to large tears; and $\geq 4$ anchors, for massive tears.

\section{Rehabilitation}

The same rehabilitation protocol was used in the tenotomy

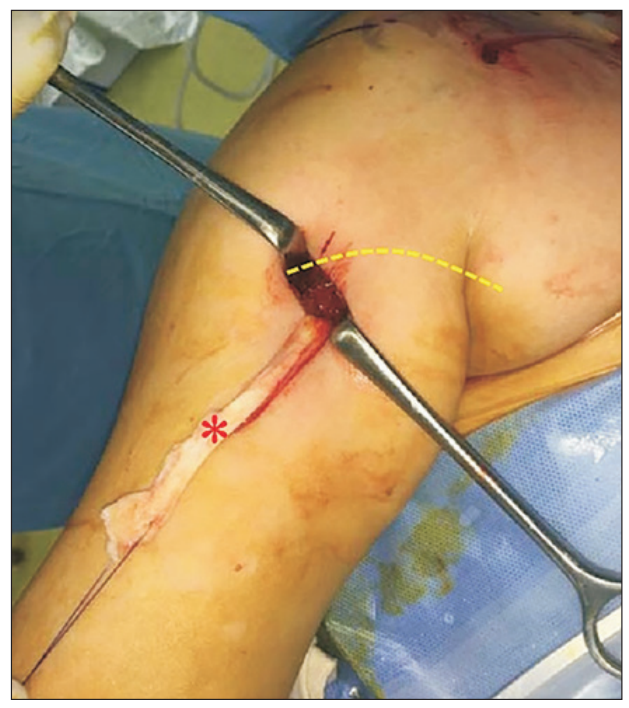

Fig. 3. The long head of the biceps tendon (asterisk) was pulled out from the bicipital groove through an incision centered at the inferior margin of the pectoralis major (dotted line: retracted pectoralis major).

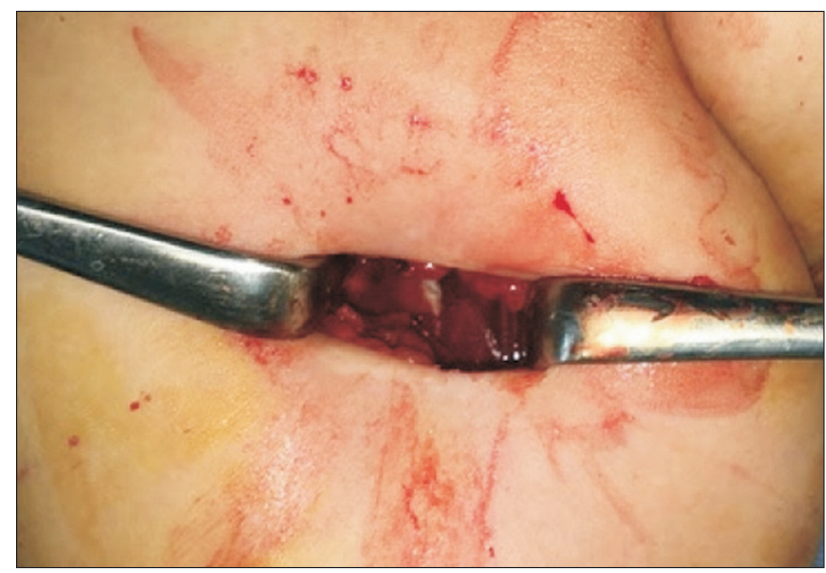

Fig. 4. Subpectoral tenodesis was completed using a soft anchor (JuggerKnot; Biomet, Warsaw, IN, USA) and sutured using a lasso-loop stitch. The remnant portion was severed.

and subpectoral tenodesis groups. Complete immobilization was maintained using an abduction brace for 5 weeks in all patients. During immobilization, elbow motions were restricted, and supination with resistance was avoided. However, hand grasp and release exercises were encouraged. After weaning from the brace, patients were instructed on how to perform passive assisted stretching exercises and active exercises in a gradual fashion (forward elevation, external rotation, and internal rotation, leading to active exercises). Return to sports was not allowed until 5 months after surgery. 


\section{Outcome Evaluation}

Functional assessment was performed using a visual analog scale (VAS) for pain, American Shoulder and Elbow Surgeons (ASES) score, Constant score, Simple Shoulder Test, and University of California Los Angeles (UCLA) shoulder score preoperatively (at admission) and at 12 months postoperatively. Shoulder activity ${ }^{15)}$ and sports activity levels were also evaluated preoperatively and at 12 months postoperatively. A clinical researcher (YEC) who was not involved in this study collected functional results of the cohorts, and the senior author (SHK) examined patients at every visit. In all included cases, postoperative cuff integrity was evaluated by MRI at 1 year postoperatively. Cases of rotator cuff healing failure (Sugaya grade 4 and 5) were excluded for better comparison of the clinical outcomes of the 2 biceps procedures.

\section{Statistical Analysis}

The analysis was performed using IBM SPSS ver. 23.0 (IBM Corp., Armonk, NY, USA). Group demographic data were compared using the Student $t$-test and the chi-square test. The Student $t$-test was used to compare outcome variables in the tenotomy and subpectoral tenodesis groups. Statistical significance was accepted for $p$-values $<0.05$.

\section{RESULTS}

The analysis was performed using the results of 77 patients who underwent either tenotomy $(\mathrm{n}=38)$ or subpectoral anchor tenodesis $(n=39)$. These 2 procedures were performed consecutively at different times. For all study subjects, the mean follow-up was $13.3 \pm 4.36$ months $(13.2 \pm 1.4$ months in the tenotomy group and $13.6 \pm 2.7$ months in the subpectoral tenodesis group; $p=0.416$ ). Indications for the biceps procedure were identical in both groups. Demographics and surgical data are summarized in Table 1 . There was no statistically significant difference in demographic characteristics between the groups. Biceps groove tenderness, Speed's test, and Yergason test were positive in $27.3 \%, 27.3 \%$, and $10.4 \%$ of the study subjects, respectively. Accompanying type II SLAP lesions, biceps subluxation, and biceps pulley lesions are listed in Table 1. No difference was evident between the 2 study groups. Postoperatively, all functional scores were significantly improved (Table 2), but no significant intergroup difference was observed even in shoulder muscle powers (all $p>0.05$ ). No patient showed a visible Popeye deformity at the final follow-up. In the tenotomy group, MRI at 1 year postoperatively revealed a tenotomized LHBT near the biceps hiatus (Fig. 5).
No patient in either group complained of arm cramping. Eight patients in the tenotomy group and 7 in the subpectoral tenodesis group complained of mild anterior shoulder pain $(p=0.731)$. Groove tenderness was noted in 4 patients in each group $(p=0.969)$. There were no surgical or postoperative complications in either group.

\section{DISCUSSION}

The main finding of this study is that tenotomy and subpectoral tenodesis conducted at the time of rotator cuff repair in patients with partial LHBT tears yielded similar clinical outcomes. The cuff healing status was evaluated by MRI at 1 year postoperatively and healing failure cases were excluded. Since biceps tenotomy or tenodesis is usually performed in conjunction with rotator cuff repair, cuff healing status would greatly affect clinical outcomes regardless of concomitant biceps procedures. In our series, failure to heal, as determined by MRI, occurred in 11 patients of the tenotomy group and in 6 patients of the subpectoral tenodesis group, and these patients were excluded from the study.

Surgeons who favor subpectoral tenodesis claim that suprapectoral tenodesis might be ineffective in improving bicipital groove tenderness because suprapectoral tenodesis might not address hidden bicipital groove pathologies, ${ }^{12,16)}$ resulting in persistent discomfort or pain. ${ }^{17,18)}$ However, it has been reported that persistent bicipital groove tenderness is common even after subpectoral tenodesis. ${ }^{19)}$ Furthermore, a recent large series study has shown that the location of tenodesis is not associated with the development of anterior shoulder pain, cramping, deformity, or subjective weakness outcomes. ${ }^{20)}$ The present study also showed that anterior shoulder pain and groove tenderness were present at similar frequencies in the subpectoral tenodesis and tenotomy groups at 1 year postoperatively. It is well known that tenotomized tendons tend to adhere to surrounding tissues including the bicipital groove. ${ }^{21)}$ Once tendon adhesion forms and no movement is anticipated at the groove, it might be reasonably expected that grooverelated biceps problems would subside. In our series, all cases in the tenotomy group had the visible biceps tendon at or near the bicipital groove entry based on MRI findings.

Biceps tenodesis may result in postoperative pain at operated sites and adversely affect patient satisfaction, ${ }^{19)}$ while such pain is not a concern for biceps tenotomy. In our series, no patient complained of incision site or anchoring site pain after subpectoral tenodesis.

Numerous studies have compared the outcomes of 
Kim et al. Tenotomy vs. Subpectoral Tenodesis

Clinics in Orthopedic Surgery • Vol. 12, No. 3, $2020 \bullet$ www.ecios.org

Table 1. Preoperative Demographics and Intraoperative Findings

\begin{tabular}{lccc}
\multicolumn{1}{c}{ Variable } & $\begin{array}{c}\text { Tenotomy group } \\
(\mathrm{n}=38)\end{array}$ & $\begin{array}{c}\text { Subpectoral tenodesis group } \\
(\mathrm{n}=39)\end{array}$ & $p$-value \\
\hline Age (yr) & $59.3 \pm 4.3$ & $58.7 \pm 5.5$ & 0.549 \\
\hline Sex (male : female) & $17: 21$ & $17: 22$ & 0.919 \\
\hline Dominant side involvement (yes : no) & $29: 9$ & $28: 11$ & 0.651 \\
\hline Biceps groove tenderness (yes : no) & $9: 29$ & $12: 27$ & 0.458 \\
\hline Speed's test (yes : no) & $13: 25$ & $8: 31$ & 0.177 \\
\hline Yergason test (yes : no) & $5: 33$ & $3: 36$ & 0.341 \\
\hline Overhead sports activity (low : medium : high) & $34: 3: 1$ & $33: 1: 5$ & 0.160 \\
\hline Shoulder activity level & $5.7 \pm 4.5$ & $5.7 \pm 5.0$ & 0.975 \\
\hline Preoperative pain VAS & $4.8 \pm 2.6$ & $4.2 \pm 2.5$ & 0.322 \\
\hline ASES score & $59.1 \pm 21.2$ & $65.8 \pm 20.3$ & 0.161 \\
\hline UCLA score & $21.3 \pm 5.7$ & $21.8 \pm 4.5$ & 0.668 \\
\hline Constant-Murley score & $62.5 \pm 16.0$ & $67.3 \pm 12.2$ & 0.112 \\
\hline Simple Shoulder Test & $4.7 \pm 1.8$ & $5.5 \pm 2.2$ & 0.123 \\
\hline Muscle power, FF (N) & $29.6 \pm 17.5$ & $32.3 \pm 17.1$ & 0.492 \\
\hline Muscle power, ER (N) & $29.3 \pm 14.0$ & $30.0 \pm 14.6$ & 0.842 \\
\hline Muscle power, IR (N) & $48.8 \pm 19.1$ & $52.9 \pm 20.6$ & 0.374 \\
\hline Acromioplasty (yes : no) & $22: 16$ & $21: 18$ & 0.721 \\
\hline Tear size (small : medium : large : massive) & $5: 21: 1: 11$ & $3: 26: 4: 6$ & 0.232 \\
\hline Type II SLAP lesion (yes : no) & $20: 18$ & $17: 22$ & 0.427 \\
\hline Biceps subluxation (yes : no) & $9: 20$ & $12: 27$ & 0.485 \\
\hline Presence of pulley lesion (yes : no) & $28: 10$ & $35: 4$ & 0.068 \\
\hline Vases a & & \\
\hline
\end{tabular}

Values are presented as mean \pm standard deviation unless otherwise indicated.

VAS: visual analog scale, ASES: American Shoulder and Elbow Surgeons, UCLA: University of California Los Angeles, FF: forward flexion, ER: external rotation, IR: internal rotation, SLAP: superior labral anterior and posterior.

biceps tenotomy and tenodesis. Biceps tenodesis is believed to preserve the normal tension-length relationship of the LHBT, to maintain elbow flexion and supination strength, and to result in less cramping pain and Popeye deformity than tenotomy. ${ }^{7,22,23)}$ By contrast, in 1 study, no outcome differences were observed even in young patients. ${ }^{24)}$ Nevertheless, some authors recommend biceps tenodesis because they consider it reduces the risk of Popeye deformity and better maintains length-tension relationships. ${ }^{14,17)}$ However, regardless of the presence of a Popeye deformity, comparative studies have usually failed to detect differences in clinical outcomes. ${ }^{9)}$

In general, comparative studies on LHBT tenotomy and tenodesis have shown that a Popeye deformity tends to occur more frequently after tenotomy. ${ }^{9,25)}$ However, in the present study, no case of Popeye deformity developed in the tenotomy group, although it should be noted that subclinical distal LHBT migration was not recognized as a Popeye deformity. Rehabilitation or the immobilization protocol after tenotomy is another important consideration. It has been well established that biceps tenotomy enables short protection and early motion. ${ }^{26)}$ On the other hand, early motion, especially active elbow motion, may contract the biceps and cause the LHBT to migrate distally. Usually, elbow motion is allowed after rotator cuff repair to prevent elbow stiffness. According to our protocol, hand grasp and release exercises were encouraged during the 5 weeks of immobilization, but elbow motion was not 

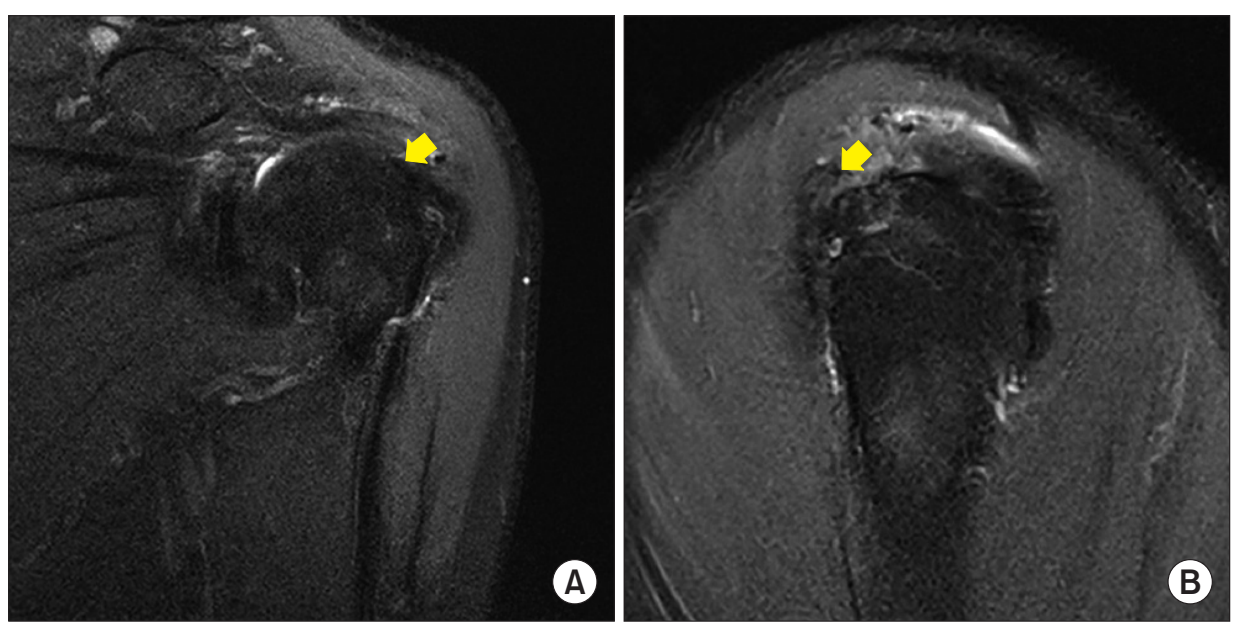

Fig. 5. Oblique coronal $(A)$ and sagittal (B) magnetic resonance imaging scans show that the tenotomized long head of the biceps tendon (arrows) remains at proximal to the bicipital hiatus.

\begin{tabular}{lccc}
\hline $\begin{array}{c}\text { Table 2. Outcomes in the Tenotomy and Subpectoral Tenodesis } \\
\text { Groups }\end{array}$ & $\begin{array}{c}\text { Tenotomy group } \\
\text { Variable }\end{array}$ & $\begin{array}{c}\text { Subpectoral } \\
\text { tenodesis group } \\
(\mathrm{n}=39)\end{array}$ & p-value \\
\hline Postoperative pain VAS & $1.1 \pm 1.8$ & $0.9 \pm 1.3$ & 0.508 \\
\hline ASES score & $91.0 \pm 11.5$ & $93.7 \pm 8.8$ & 0.260 \\
\hline UCLA score & $32.8 \pm 2.7$ & $33.5 \pm 2.3$ & 0.262 \\
\hline Constant-Murley score & $79.3 \pm 6.5$ & $81.9 \pm 7.6$ & 0.112 \\
\hline Simple Shoulder Test & $9.9 \pm 2.3$ & $10.6 \pm 2.1$ & 0.191 \\
\hline Muscle power, FF (N) & $45.0 \pm 20.8$ & $43.3 \pm 16.7$ & 0.691 \\
\hline Muscle power, ER (N) & $39.3 \pm 13.3$ & $41.5 \pm 14.5$ & 0.483 \\
\hline Muscle power, IR (N) & $64.7 \pm 23.2$ & $63.2 \pm 21.3$ & 0.762 \\
\hline
\end{tabular}

Values are presented as mean \pm standard deviation.

VAS: visual analog scale, ASES: American Shoulder and Elbow Surgeons, UCLA: University of California Los Angeles, FF: forward flexion, ER: external rotation, IR: internal rotation.

allowed. A recent study on the pullout strength of LHBT showed that considerable force is needed to dislocate the LHBT from the bicipital groove, and thus the avoidance of forceful biceps contraction during the early postoperative period may lead to an "autotenodesis effect."21) Numerous studies that evaluated the cosmetic results of tenotomy have reported that not all patients experience a Popeye deformity. In fact, the reported rates of Popeye deformity vary between $3 \%$ and $63 \%$. ${ }^{7,27,28)}$

Based on our findings, LHBT tenotomy might be a good option when performed in conjunction with rotator cuff repair for arthroscopically confirmed biceps pathology. However, we cannot conclude LHBT tenotomy would provide the same results in patients with isolated LHBT pathology without a rotator cuff tear.

This study has several limitations. First, muscle strength testing of elbow flexion and supination was not performed (forearm supination power should be measured by using a specially designed testing machine). A Popeye sign was not detected in either of the 2 study groups, but supination power may differ by method. Second, $30.4 \%$ of the candidate patients were excluded due to follow-up loss. The main reason for this was that patients refused to undergo evaluation, which included MRI, after their symptoms had subsided. Selection bias in each group would be trivial considering that the patient's condition was similar and the reason for dropout was the same. Third, it is possible that not all patients had a symptomatic biceps partial tear. The biceps procedures were undertaken based on arthroscopic findings not on preoperative biceps symptoms, and preoperative symptoms and signs of LHBT are difficult to recognize and even more difficult in the presence of a rotator cuff tear. ${ }^{29)}$ The specificities of the Speed's test and of bicipital groove tenderness for a biceps pathology in the presence of a rotator cuff tear are $45 \%$ and $57 \%$, respectively. ${ }^{30)}$ In our series, bicipital groove tenderness, Speed's test, and Yergason test were positive in $27.3 \%, 27.3 \%$, and $10.4 \%$ of the 77 study subjects, respectively. Fourth, all patients were followed up for at least 1 year postoperatively, but a longer-term follow-up is required to determine the ultimate prognoses of the 2 procedures. Fifth, we compared LHBT tenotomy and subpectoral tenodesis performed at the time of rotator cuff repair. We advise that extrapolation of our results should be performed cautiously because results may differ in younger patients with an isolated LHBT pathology, without a rotator cuff tear. Last, the sample size was relatively small. The study is underpowered due to small difference in each value with a wide standard deviation. For instance, based on the data on the 
ASES score, a sample size of 285 people should be assigned to each group to meet the statistical power of 0.8 .

Both biceps tenotomy and subpectoral tenodesis performed during rotator cuff repair improved pain and function and resulted in comparable clinical outcomes. Residual symptoms with remnant LHBT in the groove may not be a problem after adhesion of the LHBT.

\section{CONFLICT OF INTEREST}

No potential conflict of interest relevant to this article was reported.

\section{ACKNOWLEDGEMENTS}

We appreciate Young Eun Choi's (BS, Seoul National University Hospital) contribution to this study.

\section{REFERENCES}

1. Chen $\mathrm{CH}$, Hsu KY, Chen WJ, Shih CH. Incidence and severity of biceps long head tendon lesion in patients with complete rotator cuff tears. J Trauma. 2005;58(6):1189-93.

2. Habermeyer P, Magosch P, Pritsch M, Scheibel MT, Lichtenberg S. Anterosuperior impingement of the shoulder as a result of pulley lesions: a prospective arthroscopic study. J Shoulder Elbow Surg. 2004;13(1):5-12.

3. Lafosse L, Reiland Y, Baier GP, Toussaint B, Jost B. Anterior and posterior instability of the long head of the biceps tendon in rotator cuff tears: a new classification based on arthroscopic observations. Arthroscopy. 2007;23(1):73-80.

4. Oh JH, Lee YH, Kim SH, et al. Comparison of treatments for superior labrum-biceps complex lesions with concomitant rotator cuff repair: a prospective, randomized, comparative analysis of debridement, biceps tenotomy, and biceps tenodesis. Arthroscopy. 2016;32(6):958-67.

5. Park JS, Kim SH, Jung HJ, Lee $\mathrm{YH}$, Oh JH. A prospective randomized study comparing the interference screw and suture anchor techniques for biceps tenodesis. Am J Sports Med. 2017;45(2):440-8.

6. Koh KH, Kim SC, Yoo JC. Arthroscopic evaluation of subluxation of the long head of the biceps tendon and its relationship with subscapularis tears. Clin Orthop Surg. 2017;9(3):332-9.

7. Hsu AR, Ghodadra NS, Provencher MT, Lewis PB, Bach BR. Biceps tenotomy versus tenodesis: a review of clinical outcomes and biomechanical results. J Shoulder Elbow Surg. 2011;20(2):326-32.

8. Yi Y, Lee JM, Kwon SH, Kim JW. Arthroscopic proximal versus open subpectoral biceps tenodesis with arthroscopic repair of small- or medium-sized rotator cuff tears. Knee Surg Sports Traumatol Arthrosc. 2016;24(12):3772-8.

9. Castricini R, Familiari F, De Gori M, et al. Tenodesis is not superior to tenotomy in the treatment of the long head of biceps tendon lesions. Knee Surg Sports Traumatol Arthrosc. 2018;26(1):169-75.
10. Oh JH, Park MS, Rhee SM. Treatment strategy for irreparable rotator cuff tears. Clin Orthop Surg. 2018;10(2):119-34.

11. Corpus KT, Garcia GH, Liu JN, et al. Long head of biceps tendon management: a survey of the american shoulder and elbow surgeons. HSS J. 2018;14(1):34-40.

12. Rhee YG, Cho NS, Moon SC. "Hidden lesions" of the extraarticular biceps after subpectoral biceps tenodesis: response. Am J Sports Med. 2015;43(3):NP4-5.

13. Lafosse L, Van Raebroeckx A, Brzoska R. A new technique to improve tissue grip: "the lasso-loop stitch". Arthroscopy. 2006;22(11):1246.

14. Denard PJ, Dai X, Hanypsiak BT, Burkhart SS. Anatomy of the biceps tendon: implications for restoring physiological length-tension relation during biceps tenodesis with interference screw fixation. Arthroscopy. 2012;28(10):1352-8.

15. Brophy RH, Beauvais RL, Jones EC, Cordasco FA, Marx RG. Measurement of shoulder activity level. Clin Orthop Relat Res. 2005;439:101-8.

16. Moon SC, Cho NS, Rhee YG. Analysis of "hidden lesions" of the extra-articular biceps after subpectoral biceps tenodesis: the subpectoral portion as the optimal tenodesis site. Am J Sports Med. 2015;43(1):63-8.

17. David TS, Schildhorn JC. Arthroscopic suprapectoral tenodesis of the long head biceps: reproducing an anatomic length-tension relationship. Arthrosc Tech. 2012;1(1):e12732.

18. Heckman DS, Creighton RA, Romeo AA. Management of failed biceps tenodesis or tenotomy: causation and treatment. Sports Med Arthrosc Rev. 2010;18(3):173-80.

19. Millett PJ, Sanders B, Gobezie R, Braun S, Warner JJ. Interference screw vs. suture anchor fixation for open subpectoral biceps tenodesis: does it matter? BMC Musculoskelet Disord. 2008;9:121.

20. McCrum CL, Alluri RK, Batech M, Mirzayan R. Complications of biceps tenodesis based on location, fixation, and in- 
Kim et al. Tenotomy vs. Subpectoral Tenodesis

Clinics in Orthopedic Surgery • Vol. 12, No. 3, $2020 \bullet$ www.ecios.org

dication: a review of 1526 shoulders. J Shoulder Elbow Surg. 2019;28(3):461-9.

21. Kim SH, Shin SH, Oh JH, Baek GH. Biomechanical and histological analysis after tenotomy of the long head of the biceps in the rabbit shoulder model. J Orthop Res. 2012;30(3):416-22.

22. Kelly AM, Drakos MC, Fealy S, Taylor SA, O’Brien SJ. Arthroscopic release of the long head of the biceps tendon: functional outcome and clinical results. Am J Sports Med. 2005;33(2):208-13.

23. Wittstein JR, Queen R, Abbey A, Toth A, Moorman CT 3rd. Isokinetic strength, endurance, and subjective outcomes after biceps tenotomy versus tenodesis: a postoperative study. Am J Sports Med. 2011;39(4):857-65.

24. Friedman JL, FitzPatrick JL, Rylander LS, Bennett C, Vidal AF, McCarty EC. Biceps tenotomy versus tenodesis in active patients younger than 55 years: is there a difference in strength and outcomes? Orthop J Sports Med. 2015;3(2):2325967115570848.

25. Lee HJ, Jeong JY, Kim CK, Kim YS. Surgical treatment of lesions of the long head of the biceps brachii tendon with rotator cuff tear: a prospective randomized clinical trial comparing the clinical results of tenotomy and tenodesis. J Shoulder Elbow Surg. 2016;25(7):1107-14.

26. Nho SJ, Strauss EJ, Lenart BA, et al. Long head of the biceps tendinopathy: diagnosis and management. J Am Acad Orthop Surg. 2010;18(11):645-56.

27. Lim TK, Moon ES, Koh KH, Yoo JC. Patient-related factors and complications after arthroscopic tenotomy of the long head of the biceps tendon. Am J Sports Med. 2011;39(4):783-9.

28. Desai SS, Mata HK. Long head of biceps tendon pathology and results of tenotomy in full-thickness reparable rotator cuff tear. Arthroscopy. 2017;33(11):1971-6.

29. Lewis RB, Reyes BA, Khazzam MS. A review of recent advances in the diagnosis and treatment modalities for long head of bicep tendinopathy. Clin Med Insights Trauma Intensive Med. 2016;7:9-15.

30. Jain NB, Luz J, Higgins LD, et al. The diagnostic accuracy of special tests for rotator cuff tear: the ROW cohort study. Am J Phys Med Rehabil. 2017;96(3):176-83. 